\title{
AC Electrokinetics: applications for nanotechnology
}

\author{
Michael Pycraft Hughes \\ Biomedical Engineering Group \\ European Institute of Health and Medical Sciences \\ University of Surrey \\ Guildford, Surrey \\ GU2 5XH, UK
}

Telephone: (+44) 1483300800

Fax: (+44) 1483306039

Email: m.hughes@ surrey.ac.uk 


\section{Abstract}

The phenomena of dielectrophoresis and electrorotation, collectively referred to as $\mathrm{AC}$ electrokinetics, have been used for many years to study, manipulate and separate particles on the cellular (1 $\square \mathrm{m}$ or more) scale. However, the technique has much to offer the expanding field of nanotechnology, that is the precise manipulation of particles on the nanometre scale. In this paper we present the principles of $\mathrm{AC}$ electrokinetics for particle manipulation, review the current state of AC Electrokinetic techniques for the manipulation of particles on the nanometre scale, and consider how these principles may be applied to nanotechnology. 


\section{Introduction}

There are a number of key events which may be described as the origin of Nanotechnology, such as the coining of the term by Taniguchi [1974] and the publishing of Drexler's visionary "Engines of Creation" [1986]. However, the widely accepted origin of the concept of nanotechnology is the lecture "There's Plenty of Room at the Bottom" [1960] by Feynman. In this, he considered that by developing scaleable manufacturing system, a device could be made which could make a miniature replica of itself, which could in turn replicate itself in miniature, and so on down to molecular scale, a subject he revisited in the subsequent lecture "Infinitesimal machinery" [1983].

There are two principal approaches to manufacturing on the molecular scale [Drexler 1992]: selfassembly of machines from basic chemical building blocks (the "bottom up" approach) and assembly by manipulating components with much larger devices (the "top down" approach). Whilst the former is considered to be an ideal through which nanotechnology will ultimately be implemented, the latter approach is more readily achievable using current technology. Examples of this include the manipulation of single xenon atoms on a silicon surface [Eigler and Schweizer 1990] and the trapping of single 3nm colloidal particles from solution using electrostatic methods [Bezryadin et al. 1997]. In this paper, another group of methods with potential for use in top-down manufacturing - collectively referred to as AC electrokinetics - will be discussed.

AC Electrokinetic techniques such as dielectrophoresis [Jones 1995] and electrorotation [Zimmermann and Neil, 1996] have been utilised for many years for the manipulation, separation and analysis of cellular-scale particles. The phenomenon occurs due to the interaction of induced dipoles with electric fields, and can be used to exhibit a variety of motions including attraction, repulsion and rotation by changing the nature of the dynamic field. In many ways, these forces may be viewed as an electrostatic equivalent to optical tweezers [Ashkin et al. 1986] and optical 
spanners [Simpson et al. 1997] in that they exert translational and rotational forces on a body due to the interaction between a body and an imposed field gradient.

Recent advances in semiconductor manufacturing technology have enabled researchers to develop electrodes for manipulating macromolecules as small as 9kDa using both attractive [Washizu et al. 1994] and repulsive [Bakewell et al. 1998] AC electrostatic forces, and to concentrate 14nm beads from solution [Müller et al. 1996]. Trapping of single particles such as viruses and 93nm-diameter latex spheres in contactless potential energy wells [Hughes and Morgan 1998] has also been demonstrated. AC Electrokinetics offers advantages over scanning-probe methods of nanoparticle manipulation in that the equipment used is simple, cheap and has no moving parts, relying entirely on the electrostatic interactions between the particle and dynamic electric field. Furthermore, there is theoretical evidence that as manufacturing technology further improves, single particles considerably smaller than presently studied using AC Electrokinetics may be manipulated.

Ultimately, such a technology will have obvious applications for the manipulation of single molecules. Recent studies [Hughes and Morgan 1998] have shown that the trapping efficiency of planar electrode arrays is dependent on a number of factors including the magnitude and dimensions of the electric field and the radius of the particle to be trapped. In this paper we will consider the ways in which AC Electrokinetics can benefit nanotechnology, and the constraints on the technique due to factors such as Brownian motion, heating of the medium, and electrode dimensions as the electrode array is miniaturised to the nanometre scale. 


\section{Theory}

\subsection{Dielectrophoresis}

Consider a dielectric particle suspended in a spatially non-uniform electric field such as that shown in figure 1. The applied field induces a dipole in the particle; the interaction of the induced dipole with the electric field generates a force. Due to the presence of a field gradient, these forces are not equal and there is a net movement. If the particle is more polarisable than the medium around it (as shown in the figure), the dipole aligns with the field and the force acts up the field gradient towards the region of highest electric field. If the particle is less polarisable than the medium, the dipole aligns against the field and the particle is repelled from regions of high electric field [Pethig 1996]. The force is dependent on the induced dipole, and is unaffected by the direction of the electric field, responding only to the field gradient. Since the alignment of the field is irrelevant, this force can also be generated in AC fields which has the advantage of reducing any electrophoretic force (due to any net particle charge) to zero.

This effect was first termed Dielectrophoresis by Pohl [1978]. The dielectrophoretic force, $F_{D E P}$, acting on a spherical body is given by:

$$
F_{D E P}=2 \pi r^{3} \varepsilon_{m} \operatorname{Re}[K(\omega)] \nabla E^{2}
$$

where $r$ is the particle radius, $\varepsilon_{m}$ is the permittivity of the suspending medium, $\nabla$ is the Del vector operator, $E$ is the rms. electric field and $\operatorname{Re}[K(\omega)]$ the real part of the Clausius-Mossotti factor, given by:

$$
K(\omega)=\frac{\varepsilon_{p}^{*}-\varepsilon_{m}^{*}}{\varepsilon_{p}^{*}+2 \varepsilon_{m}^{*}}
$$


where $\varepsilon_{m}{ }^{*}$ and $\varepsilon_{p}{ }^{*}$ are the complex permittivities of the medium and particle respectively, and $\varepsilon^{*}=\varepsilon-\frac{\mathrm{j} \sigma}{\omega}$ with $\sigma$ the conductivity, $\varepsilon$ the permittivity and $\omega$ the angular frequency of the applied electric field.

The frequency-dependence of $\operatorname{Re}[K(\omega)]$ indicates that the force acting on the particle varies with the frequency. The magnitude of $\operatorname{Re}[K(\omega)]$ also varies depending on whether the particle is more or less polarisable than the medium. If $\operatorname{Re}[K(\omega)]$ is positive, then particles move to regions of highest field strength (positive dielectrophoresis); the converse is negative dielectrophoresis where particles are repelled from these regions. By careful construction of the electrode geometry which creates the electric field, it is possible to create electric field morphologies so that potential energy minima are bounded by regions of increasing electric field strengths. In such electrodes, particles experiencing positive dielectrophoresis are attracted to the regions of highest electric field (typically the electrode edges, particularly where adjacent electrodes are close), whilst particles experiencing negative dielectrophoresis are trapped in isolated field minima.

A phenomenon often observed in dielectrophoresis experiments is electro-orientation [e.g. Miller and Jones 1993]. When a non-spherical object is suspended in an electric field (for example, but not solely, when experiencing dielectrophoresis) it rotates such that the dipole along the longest non-dispersed axis aligns with the field. Since each axis has a different dispersion, the particle orientation will vary according to the applied frequency. For example, at lower frequencies, a rodshaped particle experiencing positive dielectrophoresis will align with its longest axis along the direction of electric field. As the frequency is increased, the dipole along this axis reaches dispersion, but the dipole formed across the rod does not and the particle will rotate $90^{\circ}$ and align 
perpendicular to the field. This alignment force may also be observed in the electrorotation of elliptical objects, for example latex beads, which can lead to object exhibiting a "wobble" when rotating.

\subsection{Electrorotation}

If a polarisable particle is suspended in a rotating electric field, the induced dipole will form across the particle and should rotate in synchrony with the field. However, if the angular velocity of the field is sufficiently large, the time taken for the dipole to form (the relaxation time of the dipole) becomes significant and the dipole will lag behind the field. This results in non-zero angle between field and dipole, which induces a torque in the body and causes it to rotate asynchronously with the field; the rotation can be with or against the direction of rotation of the field, depending of whether the lag is less or more than $180^{\circ}$. This is shown schematically in figure 2 . The general equation for time-averaged torque $\Gamma$ experienced by a spherical polarisable particle of radius $r$ suspended in a rotating electric field $\mathrm{E}$ is given by:

$$
\Gamma=-4 \pi \varepsilon_{\mathrm{m}} \mathrm{r}^{3} \operatorname{Im}[K(\omega)] \mathrm{E}^{2}
$$

where $\operatorname{Im}[K(\square)]$ represents the imaginary component of the Clausius-Mossotti factor. The minus sign indicates that the dipole moment lags the electric field. When viscous drag is accounted for, the rotation rate $\mathbf{R}(\square)$ of the particle is given by [Arnold and Zimmerman 1988]:

$$
\mathbf{R}(\omega)=-\frac{\varepsilon_{m} \operatorname{Im}[K(\omega)] \mathbf{E}^{2}}{2 \eta}
$$

Note that there are two significant differences between the expression for torque as shown in equation (3) and the force in equation (1). Firstly, the relationship with the electric field is as a 
function of the square of the electric field rather than of the gradient of the square of the electric field. Secondly, the torque depends on the imaginary rather than the real part of the ClausiusMossotti factor. This is significant in that a particle may experience both dielectrophoresis and electrorotation simultaneously, and the magnitudes and directions of both are related to the interaction between the dielectric properties of particle and medium. As an example of this, a plot of the frequency-dependence of a solid particle exhibiting a single dielectric dispersion is shown in figure 3 .

\subsection{Travelling-Wave Dielectrophoresis}

Travelling-wave dielectrophoresis is a linear analogue of electrorotation; instead of electrodes being arranged in a circle, they are laid out as "tracks", but the relationship of phases (each electrode being $90^{\circ}$ phase advanced from the last) remains. This produces an electric field wave which "travels" along the electrodes [Hughes et al. 1996]. When this wave interacts with a polarisable particle, a dipole is induced which moved with the electric field peak. If the travelling wave is moving sufficiently quickly, the dipole will lag behind the field in a similar manner to that observed in electrorotation. However, as the wave is travelling in a linear (rather than rotational) manner, the result is the induction of a force rather than a torque, with particles moving along the electrodes like a train travelling along tracks. This is demonstrated schematically in figure 4; the value of the force $F_{T W D}$ is given by equation (5) [Huang et al. 1992]:

$$
F_{T W D}=\frac{-4 \pi \varepsilon_{m} r^{3} \operatorname{Im}[K(\omega)] E^{2}}{\lambda}
$$

where $\square$ is the wavelength of the travelling wave. This phenomenon was first observed by Batchelder [1983]. However, it remained largely unexplored until work by Masuda et al. [1987] on using synchronously induced motion in low frequency travelling fields that travelling wave 
dielectrophoresis became significant. This work led to Fuhr et al. [1991] effectively rediscovering asynchronous travelling wave dielectrophoresis. Since then, a large corpus of study has been conducted on this technique, including theoretical studies (Huang et al. [1993]; Hughes et al. [1996]) and demonstrations of practical devices for electrostatic pumping (e.g. Fuhr et al. 1994) and biotechnological applications (e.g. Morgan et al. [1997])

\subsection{Electrode configurations}

In practice, dielectrophoretic manipulation of particles is performed on planar electrode arrays. Such electrode arrays employ patterned electrodes fabricated of gold or a similar conductor deposited on glass or silicon. A common electrode configuration used in AC Electrokinetics research is a quadrupole arrangement where four electrodes point towards a central enclosed region [Huang and Pethig 1991], as shown in the schematic in figure 5. These electrodes may be of a variety of designs, according to the degree of field non-uniformity required [Hughes 1998]. The advantage with this arrangement is twofold. Firstly, electrodes can easily be used for dielectrophoresis, electrorotation or both. By energising the electrodes such that the phase angle of applied AC field between adjacent electrodes is $180^{\circ}$, particles will experience only dielectrophoresis. If the phase angle is reduced such that adjacent electrodes differ by $90^{\circ}$, the particle will experience both electrorotation and dielectrophoresis. The second advantage of the quadrupole design is that at the centre of the electrode array, there exists a well-defined electric field minimum, which is surrounded by well-defined field maxima at the electrode edges. This definition is advantageous when attempting to manipulate nanometre-sized particles, where Brownian motion is significant and may be sufficient to enable particles to "escape" from a weak forcefield "trap". This is illustrated in figure 6, which shows a simulation of the electric field in a plane $5 \square \mathrm{m}$ above the plane of the electrodes shown in figure 5. The dark circular region at the centre of the electrode array is the field minimum in which particles become trapped by the 
surrounding ring of high field gradient. The field maximum extends along the inter-electrode gaps, where particles experiencing positive dielectrophoresis will accumulate.

Advances in fabrication technology such as the use of electron beam lithography have allowed the manufacture of electrodes with feature sizes of the order $100 \mathrm{~nm}$, covering many square $\mathrm{cm}$. By reducing the size of inter-electrode gaps, electric fields of $10^{7} \mathrm{Vm}^{-1}$ can be generated by applying 10V across a $1 \square \mathrm{m}$ inter-electrode gap. More advanced dielectrophoretic traps have been developed [Schnelle et al. 1993] in which two sets of electrodes were stacked so as to form an octopole field "cage". This type of geometry has allowed the controlled manipulation of populations of particles in all 3 dimensions.

Other electrode configurations have been demonstrated to be effective for nanoparticle manipulation. For example, Rousselet et al. [1994] demonstrated a "Christmas tree" electrode structure - effectively a thermal ratchet - which was used to pump 250nm-diameter latex beads. Another, more complex structure has recently been reported by Suehiro and Pethig [1998]. The structure consisted of electrode arrays placed above and below the particle suspension. The arrays comprised several flat parallel wires arranged so that the wires in the top array were at $90^{\circ}$ to those in the bottom array. By careful control of the potentials applied to individual electrode arrays, it was shown that single protoplasts (diameter $30 \square \mathrm{m}$ ) could be "steered" around the array. Whilst this technique has not yet been demonstrated using single nanometre-scale particles, the manipulation of such particles using such an array should be readily achievable. 


\section{Applications to Nanotechnology}

\subsection{Concentration of colloids from solution}

Since the force experienced by a particle undergoing dielectrophoresis scales as a function of the particle volume, it was believed for many years that a lower threshold of particle size existed, below which the dielectrophoretic force would be overcome by Brownian motion. It was held that to increase the force would require electric fields of such magnitude that local medium heating would increase local fluid flow, again acting to prevent dielectrophoretic manipulation. As electrode fabrication techniques were relatively crude, generating electric fields of sufficient non-uniformity required very large potentials to be applied across relatively large inter-electrode volumes, and consequently particles with diameters less than about $1 \square \mathrm{m}$ could not be trapped. Indeed, Pohl [1978] speculated that particles smaller 500nm would require excessively large electric fields to trap against the action of Brownian motion.

The first group to break this threshold was that of Washizu and co-workers [1994] who used positive dielectrophoresis to precipitate DNA and proteins as small as $25 \mathrm{kDa}$. This step downward in size was accelerated by improvements in technologies for electrode fabrication, principally the use of electron beam fabrication. This renewed interest in manipulation of sub-micron particles, and subsequent work by Fuhr and co-workers [Fuhr 1995; Müller et al. 1996] and Green et al. [1995, 1997] demonstrated that viruses of $100 \mathrm{~nm}$ diameter could be manipulated using negative dielectrophoresis. It was also demonstrated that latex spheres of $14 \mathrm{~nm}$ diameter could be trapped by either positive or negative dielectrophoresis, as demonstrated by Müller et al. [1996] and reproduced here in figure 7. Subsequent work by Hughes and co-workers demonstrated that by varying the frequency of the applied electric field, Herpes viruses can be trapped using either positive or negative electric fields [1998]. Another study demonstrated that molecules of the 
68kDa-protein avidin can be concentrated from solution by both positive and negative dielectrophoresis [Bakewell et al. 1998].

The most obvious nanotechnological application of this technique is in the concentrating of "parts" of molecular machinery to one site. This could be either in the form of bringing together of two interlocking molecules in a highly dilute solution, or collecting "fuel" for a nanomachine from solution. Furthermore, by attracting microspheres and colloidal gold particles which have been functionalised using antibodies, Velev and Kaler [1999] have used dielectrophoresis to construct microscopic biosensors by "stacking" particles of different types, which bind on contact.

\subsection{Manipulation of single nanoparticles.}

The first report of molecular-scale particle manipulation was that of Washizu and Kurosawa in 1990. In this paper, DNA fragments of 48.5kilobase pairs (approximately $16 \square \mathrm{m}$ ) long were trapped and stretched by positive dielectrophoresis, and held whilst a UV source was used to "cut" the fragment. It was also shown that fragments of different lengths could be sorted into ascending order of size, stretched across an inter-electrode gap. Later work [Washizu et al. 1994a] demonstrated the same technique with smaller DNA fragments and a laser, and to collect the cut fragments. This work demonstrated that the manipulation of single molecular-scale objects (albeit relatively large molecules) could be manipulated and, in conjunction with the same group's work on proteins [Washizu et al. 1994], formed the foundations of sub-micrometre dielectrophoresis research.

In 1996, Müller et al. Demonstrated that a single $1 \square \mathrm{m}$ latex sphere could be trapped by negative dielectrophoresis, and subsequently repeated the experiment with a latex sphere of $650 \mathrm{~nm}$ diameter. Further work by Hughes and Morgan [1998] demonstrated that single Herpes viruses, viral capsids and latex spheres as small as $93 \mathrm{~nm}$ diameter could be held in stable traps using negative 
dielectrophoresis, as shown in figure 8. This study also considered in some detail the mathematical conditions for a particle to be trapped by either positive or negative dielectrophoresis, concluding that for stable trapping, an approximate minimum particle radius $r$ is given by the expression:

$$
r>\sqrt[3]{\frac{10 k T}{\pi \varepsilon_{m} \Delta d \operatorname{Re}[K(\omega)] \nabla E^{2}}}
$$

where $\mathrm{k}$ is Bolzmann's constant, $\mathrm{T}$ is the temperature, and $\Delta d$ is the thickness of the electric field barrier of magnitude, $\nabla E^{2}$. Since the factors $\Delta d$ (related to the electrode geometry) and $\nabla E^{2}$ are related to the size of the electrode array, they place limits on the minimum size of particle an array can be used to manipulate. Studies by Hughes and Morgan [1998] indicated that for electrode structures with $5 \mathrm{Vp}$-p applied to electrodes with $6 \square \mathrm{m}$ between opposing electrodes and $2 \square \mathrm{m}$ between adjacent electrodes, the minimum stable trapping radius for single particle is approximately $30 \mathrm{~nm}$. This research indicated that the key variable governing $\Delta d$ is the size of the centre of the trap, which in that research was an area of approximately $5 \square \mathrm{m}$ radius centred on the middle of the electrodes. Therefore it should theoretically be possible to reduce the whole array to this size; this would benefit the use of this technique in nanotechnology since the manipulator would be of the order of a few microns across. Furthermore, since $\Delta d$ scales linearly with distance and $\nabla E^{2}$ scales as the inverse cube of distance, equation (5) indicates that reducing the size of the trap by a factor $x$ whilst maintaining the same activating potentials will increase the efficiency of the trap by reducing the minimum trappable particle radius by a factor $\mathrm{x}^{2 / 3}$.

Due to the fact that this trapping is contactless, with particles being repelled from the electrode edges, particles may move upwards and will eventually leave the trap. In order to prevent this happening closed field cages, such as those fabricated by Schnelle et al. [1996] must be employed. An octopole cage of sufficiently small dimension could in theory allow full 3D manipulation of 
single particles of less than $30 \mathrm{~nm}$ diameter, and has been demonstrated to function for aggregates of 14nm particles. The technology to produce such electrodes for single-molecule manipulation exists, but the principal problem to be overcome is the observation of the trapped particle.

An alternative approach to single particle manipulation would be to use an AFM tip to generate field non-uniformity, and use positive dielectrophoresis to attract particles to it. These could be positioned by the AFM and then "released" by changing the frequency of the signal to the AFM tip so as to repel the particles from it. The principle behind this - using positive dielectrophoresis to attract particles to a 3D, microscopic needle tip - has already been demonstrated [Alimova et al. 1999] to coat silicon tips with nanodiamond.

\subsection{Particle separation}

In 1992 Gascoyne et al. demonstrated that positive and negative dielectrophoresis could be used to separate a heterogeneous population of normal and leukaemic murine erythrocytes into two separate populations. Since the direction of the induced dielectrophoretic force is determined by the dielectric properties of the particle, populations of particles having different dielectric properties can be induced to move in different directions by careful choice of applied field frequency and medium conductivity. Since then a broad range of particle separation techniques have been demonstrated on cells including yeast, cancer cells and bacteria [e.g. Markx et al. 1994; Becker et al. 1994; Markx and Pethig 1994] among many others. More recently, examples of this work in the submicrometre range have been demonstrated by Morgan et al. [1999] in the separation of a mixture of Herpes Simplex and Tobacco Mosaic viruses into two distinct populations, and the separation of latex spheres of different sizes, or of similar size but different surface treatments. Another example of this is the separation of $93 \mathrm{~nm}$ diameter latex spheres according to small variations in the surface charge of the spheres, as demonstrated by Green and Morgan [1997]. 
In a nanotechnological context, the dielectrophoretic separation of particles allows for the manufacture of a tuneable electrostatic filter. By altering the frequency of the applied electric field, particles for two populations can be either both attracted to the electrode array, both repelled from it, or one can be attracted whilst the other is repelled. Since the effect is volumetric - repulsion acts for several microns into the solution, according to the magnitude of the electric field - the result is to alter the local concentration of the two particle types dramatically.

\subsection{Particle Transport}

Whilst dielectrophoresis is useful for attracting particles to specific points, there are applications which require the moving of particles between one position and another. This requirement may be to relocate particles for processing, for separation purposes, or to gather in particles from a larger volume than can be achieved by conventional dielectrophoresis alone. Two means of particle transport have been demonstrated in the literature. Firstly, conventional dielectrophoresis has been used to move $100 \mathrm{~nm}$ latex spheres in a thermal ratchet, where dielectrophoresis is used to rectify the motion of particles induced by Brownian motion [Rousselet et al. 1994]. The induced motion can be either towards or against the ratchet potential, according to whether particles experience positive or negative dielectrophoresis, or the time between activation of ratcheting pulses [Chauwin et al. 1995]. This technique has the advantage that since only two phases are required, the electrodes for inducing such motion are easy to fabricate.

The alternative, and more popular, means of transporting particles using AC electrokinetics is by the application of travelling-wave dielectrophoresis. Whilst the majority of work on travellingwave dielectrophoresis has concerned micrometre-sized objects such as blood cells [Morgan et al. 1997], some work has been performed on the concentration of particles on a surface using so-called "meander" electrodes [Fuhr et al. 1994b]. These structures use four electrodes in a series of interlocking spirals to generate a travelling wave; at the centre of the spiral, the electrodes form a 
quadrupole-type electrode array. It has been demonstrated that by careful manipulation of the amplitudes of the potentials on these electrode structures, it is possible to "steer" the motion of particles across the array. Tools such as these could be used as the basis for "conveyor belts" for factories on a chip, wherein different chemical processes may be carried out of the same chip, with operations performed by electrostatic or chemical means and the resultant output transferred to a new process by AC Electrokinetic means [Ward 1997].

\subsection{Electrorotation}

Electrorotation was first examined scientifically in the early 1960s [e.g. Teixeira-Pinto et al. 1960] but was most fully explored by Arnold and Zimmermann [1982; 1988], who established the technique for direct measurement of the value of $K(\square)$ by measuring the time taken for a particle to rotate. This application has little bearing to nanotechnology, not least due to the difficulty of seeing such small particles rotate, but electrorotation may be useful as a means of inducing rotation in molecular machinery. Recent work by Berry and Berg [1999] has involved using electrorotation to drive the molecular motor of $E$. coli bacteria backwards at speeds of up to $2,000 \mathrm{~Hz}$. Since the electrodes involved in that work were applying $10 \mathrm{~V}$ across inter-electrode gaps of $50 \square \mathrm{m}$ or more, considerably higher fields (and hence induced torques) could be applied with electrodes with interelectrode gaps of the order of $1 \square \mathrm{m}$. If one considers that electrorotation is an electrostatic equivalent of laser spanners [Simpson et al. 1997], then there exists a number of applications within current nanotechnological thought to which electrorotation might be applied. There are a number of advantages that favour electrorotation over its optical equivalent; most notable advantages are that electrorotation-induced torque is easily controlled by altering the frequency of the electric field, and that there does not need to be a direct optical path to the part to be manipulated. Electrorotation has been demonstrated for the induction of torque in micron-scale motors by Hagedorn et al. [1994]. 


\subsection{Brownian motion, conduction and convection}

Aside from the engineering of electrodes small enough to generate large electric field nonuniformity, there are other factors that present problems with scaling AC Electrokinetic techniques from the micrometre to nanometre scale. These include Brownian motion induced by thermal noise in the surrounding medium and effects of conduction and convection of the medium induced by the high electric fields used for manipulating colloidal particles. For sufficiently high concentrations of particles, a diffusion force would also be significant; however, the majority of work on dielectrophoresis has used sufficiently low concentrations of particles for each particle to be regarded as single and isolated.

Brownian motion due to thermal noise is well documented [Einstein 1956] and a number of studies have considered the force required to trap particles against Brownian motion [Pohl 1978; Washizu et al. 1994; Hughes and Morgan 1998]. Experimental studies with proteins [Washizu et al. 1994; Bakewell et al. 1998] have demonstrated that Brownian motion does not prevent the trapping particles as small as $68 \mathrm{kDa}$ (equivalent to a cube $6 \mathrm{~nm}$ along a face) by negative dielectrophoresis, or 25kDa by positive dielectrophoresis. Recent studies (Ramos et al. [1998] Green et al. [1999]) on the forces involved in dielectrophoretic manipulation of sub-micrometre particles examined the balance of Brownian motion and dielectrophoretic forces by considering the amount of time required to determine, with $99.7 \%$ confidence (3 standard deviations) that an observed force is due to dielectrophoresis. Any force applied to a particle will ultimately result in what Ramos et al. [1998] describes as "observable deterministic motion", but if the force is small the time taken to observe it may be of the order of years! However, the forces used in the experiments described in the literature are sufficiently large for the applied force to observably overcome Brownian motion to be of the order of a second or (often significantly) less. It may ultimately be possible to manipulate single small particles precisely using dielectrophoretic forces, but this will be more difficult to achieve for very small colloids and work needs to be done to study this. 
Other disruptive effects are caused by the presence of very high electric fields covering small areas of the electrode array, creating electrohydrodynamic (EHD) motion in the fluid. An in-depth study of this subject has been conducted by Ramos et al. [1998], and presented in summary form by Green et al. [1999]. In these works, the authors demonstrated that there are two major EHD forces which are significant in driving fluid flow around electrodes, those being electrothermal and electroosmotic. The former force is due to localised heating of the medium causing discontinuities of medium conductivity and permittivity, which was found to be insignificant due to the small volumes these discontinities occupy (as the electrodes are so small). The latter force is due to the interaction of the tangential electric field with the diffuse double layer above the electrode surfaces. This creates a fluid pumping action, the magnitude of which can be several orders of magnitude larger than the dielectrophoretic force. However, careful electrode design can allow the EHD forces to be used as an aid to particle manipulation [Green and Morgan 1998].

\section{Conclusion}

The techniques of AC Electrokinetics, principally dielectrophoresis and electrorotation, have much to offer the expanding science of nanotechnology. Whereas dielectrophoretic methods have been applied to the manipulation of objects in the micro-scale, recent work shows that the technique is applicable to the manipulation of nano-scale particles. This opens a wide range of potential applications for AC electrokinetics to the development of mainstream nanotechnology.

The fundamental challenge in the advancement of nanotechnology is the development of precision tools for the manipulation of macromolecules in solution phase. The techniques presented in this paper go some way to addressing this by providing tools for the trapping, manipulation and separation of biomolecules and small latex spheres using tools (electrodes) on the micrometre scale. 
There exist a number of avenues down which AC electrokinetics can be developed to meet the needs of nanotechnology more readily. These include the design of electrodes for trapping of smaller single particles, increasing the precision to which particles may be trapped, and examining the extent to which particles may be manoeuvred within the trap by alteration of the electric field.

\section{Acknowledgements}

The author would like to thank Dr. Hywel Morgan of the University of Glasgow and Dr Thomas Schnelle of the Humboldt University of Berlin for the experimental photographs presented in this paper, and Dr. Nicolas Green of the University of Seville for valuable discussions. 


\section{References}

Alimova AN, Chubun NN, Belobrov PI, Ya Detkov P, Zhirnov VV (1999) J. Vac. Sci. Technol. Vol. 17 pages 715-718. Electrophoresis of nanodiamond powder for cold cathode fabrication.

Arnold WM, Zimmermann U (1982) Z. Naturforsch. Vol. 37c, pages 908-915. Rotating-fieldinduced rotation and measurement of the mambrane capacitance of single mesophyll cells of Avena sativa.

Arnold WM, Zimmermann U (1988) J. Electrostatics Vol. 21 pages 151-191. Electro-rotation development of a technique for dielectric measurements on individual cells and particles.

Ashkin A, Dziedzic JM, Bjorkholm JE, Chu S (1986) Optics letters Vol. 11 pages 288-290. Observation of a single-beam gradient force optical trap for dielectric particles.

Bakewell DJG, Hughes MP, Milner JJ, Morgan H (1998) Proc. 20th Annual International Conference of the IEEE Engineering in Medicine and Biology Society. Dielectrophoretic manipulation of Avidin and DNA

Batchelder JS (1983) Rev. Sci. Instrum Vol. 54 pages 300-302. Dielectrophoretic manipulator.

Becker FF, Wang X-B, Huang Y, Pethig R, Vykoukal J, Gascoyne PRC (1994) J. Phys. D: Appl. Phys Vol. 27 pages 2659-2662. The removal of human leukaemia cells from blood using interdigitated microelectrodes. 
Berry RM, Berg HC (1999) Biophys. J. Vol. 76 pages 580-587. Torque generated by the flagellar motor of Escherichia coli while driven backward.

Bezryadin A, Dekker C, Schmid G (1997) Appl. Phys. Lett. Vol. 71 pages 1273-1275. Electrostatic trapping of single conducting nanoparticles between nanoelectrodes.

Chauwin J-F, Ajdari A, Prost J (1995) Europhys. Lett. Vol. 32 pages 373-378. Current reversal in asymmetric pumping.

Drexler, KE (1986) Engines of Creation. New York: Anchor books/Doubleday.

Drexler, KE (1992) Nanosystems; molecular machinery, manufacturing and computation. New York: Wiley.

Eigler DM, Schweizer EK (1990) Nature Vol. 344 page 524-526. Positioning single atoms with a scanning tunnelling microscope.

Einstein A (1956) Investigations on the Theory of Brownian Movement. New York: Dover.

Feynman R (1960) reprinted in J. Microelectromechanical systems (1992) Vol. 1 pages 60-66. There's plenty of room at the bottom.

Feynman R (1983) reprinted in J. Micromechanical Systems (1993) Vol. 2 pages 4-14. Infinitesimal machinery. 
Fuhr G, Hagedorn R, Müller T, Benecke W, Wagner B, Gimsa J (1991) Studia Biophysica Vol. 140 pages 79-102. Asynchronous traveling-wave induced linear motion of living cells.

Fuhr G, Schnelle T, Wagner B (1994) J. Micromech. Microeng. Vol. 4 pages 217-226. Travellingwave driven microfabricated electrohydrodynamic pumps for liquids.

Fuhr G, Fiedler S, Müller T, Schnelle T, Glasser H, Lisec T, Wagner B (1994b) Sensors and Actuators A Vol. 41-42 pages 230-239. Particle micromanipulator consisting of two orthogonal channels with travelling-wave electrode structures.

Fuhr, G (1995) Proc. St Andrews Meeting of the Society for Experimental Biology (St Andrews, UK) page 77.

Gascoyne PRC, Huang Y, Pethig R, Vykoukal J, Becker FF (1992) Meas. Sci. Technol. Vol. 3 pages 439-445. Dielectrophoretic separation of mammalian cells studied by computerised image analysis.

Green NG, Morgan H, Wilkinson CDW, Milner JJ (1995) Proc. St Andrews Meeting of the Society for Experimental Biology (St Andrews, UK) page 77. Dielectrophoresis of virus particles.

Green NG, Morgan H (1997) J. Phys. D: Appl. Phys. Vol 30 pages L41-L44. Dielectrophoretic separation of nano-particles.

Green NG, Morgan H and Milner JJ (1997) J. Biochem Biophys. Methods Vol. 35 pages 89-102. Manipulation and trapping of sub-micron bioparticles using dielectrophoresis 
Green NG, Morgan H (1998) J. Phys. D: Apply. Phys. Vol.31 pages L25-L30. Separation of submicrometre particles using a combination of dielectrophoretic and electrohydrodynamic forces

Green NG, Ramos A, Morgan H, Castellanos A (1999) Inst. Phys. Conf. Ser. Vol. 163 pages 89-92. Sub-micrometre AC electrokinetics: particle dynamics under the influence of dielectrophoresis and electrohydrodynamics.

Hagedorn R, Fuhr G, Müller T, Schnelle T, Schnakenberg U, Wagner B (1994) J. Electrostatics Vol. 33 pages 159-185. Design of asynchronous dielectric micromotors.

Huang Y, Pethig R (1991) Meas. Sci. Technol. Vol. 2 pages 1142-1146. Electrode design for negative dielectrophoresis.

Huang Y, Wang X-B, Tame J, Pethig R (1993) J. Phys. D: Appl. Phys. Vol 26 pages 312-322. Electrokinetic behaviour of colloidal particles in travelling electric fields: studies using yeast cells.

Hughes MP, Pethig R, Wang X-B (1996) J. Phys. D: Appl. Phys. Vol. 29 pages 474-482. Forces on Particles in Travelling Electric Fields: Computer-Aided Simulations

Hughes MP, Morgan H, Rixon FJ, Burt JPH, Pethig R (1998) Biochima et Biophysica Acta Vol. 1425 pages 119-126. Manipulation of Herpes Simplex Virus type 1 by Dielectrophoresis.

Hughes MP (1998) Phys. Med. Biol. Vol. 12 pages 3639-3648. Computer-aided analysis of Conditions for Optimising Practical Electrorotation 
Hughes MP, Morgan H (1998) J. Phys. D: Appl. Phys. Vol. 31 pages 2205-2210. Dielectrophoretic Manipulation of Single Sub-micron Scale Bioparticles

Hughes MP, Archer S, Morgan H (1999) J. Phys. D: Appl. Phys. Vol 32 pages1548-1552. Mapping the electrorotational torque in planar microelectrodes.

Jones, TB (1995) Electromechanics of particles. Cambridge: Cambridge University Press.

Markx GH, Talary MS, Pethig R (1994) J. Biotechnol. Vol. 32 pages 29-37. Separation of viable and non-viable yeast using dielectrophoresis.

Markx GH, Pethig R (1994) Biotechnology and Bioengineering Vol. 45 pages 337-343.

Dielectrophoretic separation of cells: continuous separation.

Masuda S, Washizu M, Iwadare M (1987) IEEE Trans. Ind. Appl. Vol. 23 pages 474-480. Separation of small particles suspended in liquid by nonuniform traveling field.

Miller RD, Jones TB (1993) Biophys. J. Vol. 64 pages 1588-1595. Electro-orientation of ellipsoidal erythrocytes.

Morgan H, Green NG, Hughes MP, Monaghan W, Tan TC (1997) J. Micromech. Microeng. Vol. 7 pages 65-70. Large-area travelling-wave dielectrophoresis particle separator.

Morgan H, Hughes MP, Green NG (1999) Biophys. J. Vol. 77 pages 516-525. Separation of SubMicron Particles by Dielectrophoresis 
Müller T, Gerardino A, Schnelle T, Shirley SG, Bordoni F, DeGasperis G, Leoni R, Fuhr G (1996)

J. Phys. D: Appl. Phys. Vol 29 pages 340-349. Trapping of micrometre and sub-micrometre particles by high-frequency electric fields and hydrodynamic forces.

Pethig R (1996) Critical Reviews in Biotechnology Vol. 16 pages 331-348. Dielectrophoresis: using inhomogeneous AC electrical fields to separate and manipulate cells.

Pohl HA (1978) Dielectrophoresis. Cambridge: Cambridge University Press.

Ramos A, Morgan H, Green NG, Castellanos A (1998) Vol. 31 pages 2338-2353. AC electrokinetics: a review of forces in microelectrode structures.

Rousselet J, salome L, Ajdari A, Prost J (1994) Nature Vol. 370 pages446-448. Directional motion of Brownian particles induced by a periodic asymmetric potential.

Schnelle T, Hagedorn R, Fuhr G, Fiedler S, Müller T (1993) Biochim. Biophys. Acta Vol. 1157 pages 127-140. Three-dimensional electric field traps for manipulation of cells - calculation and experimental verification.

Schnelle T, Müller T, Fiedler S, Shirley SG, Ludwig K, Hermann A, Fuhr G (1996) Naturwissenschaften Vo. 83, pages 172-176. Trapping of viruses in high-frequency electric field cages.

Simpson NB, Dholakia K, Allen L, Padgett MJ (1997) Optics Letters Vol. 22 pages 52-54. Mechanical equivalence of spin and orbital angular momentum of light: an optical spanner. 
Suehiro J, Pethig R (1998) J. Phys. D: Appl. Phys. Vol. 31 pages 3298-3305. The dielectrophoretic movement and positioning of a biological cell using a three-dimensional grid electrode system.

Taniguchi N (1974) Proc. Int. Conf. Prod. Eng.. Page 18. On the basic concept of nanotechnology.

Teixeira-Pinto AA, Nejelski LL, Cutler JL, Heller JH (1960) J. Exp. Cell Res. Vol.20 pages 548564. The behaviour of unicellular organisms in an electromagnetic field.

Velev OD, Kaler EW (1999) Langmuir Vol. 15 pages 3693-2698 . In situ assembly of colloidal particles into miniaturised biosensors

Ward M (1997) New Scientist, $1^{\text {st }}$ March, pages 22-26. Devilish tricks with tiny chips.

Washizu M, Kurosawa O (1990) IEEE Trans. Ind. Appl. Vol. 26 pages 1165-1172. Electrostatic manipulation of DNA in microfabricated structures.

Washizu M, Suzuki S, Kurosawa O, Nishizaka T, Shinohara T (1994) IEEE Trans. Ind. Appl. Vol. 30 pages 835-842. Molecular dielectrophoresis of biopolymers.

Washizu M, Kurosawa O, Arai I, Suzuki S,Shimamato N (1994a) IEEE Trans. Ind. Appl. Vol. 31 pages 447-456. Applications of electrostatic stretch-and positioning of DNA.

Zimmermann U, Neil GA (1996) Electromanipulation of cells. CRC press. 


\section{Figure Legends}

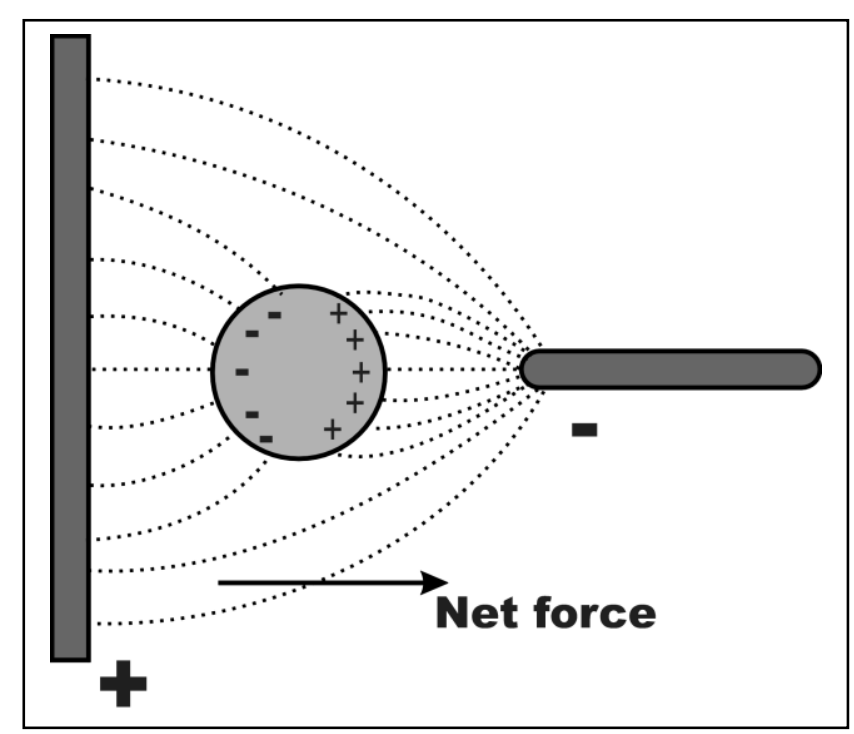

Figure 1. A schematic of a polarisable particle suspended within a point-plane electrode system. When the particle polarises, the interaction between the dipolar charges with the local electric field produces a force. Due to the inhomogeneous nature of the electric field, the force is greater in the side facing the point than that on the side facing the plane, and there is net motion towards the point electrode. This effect is called positive dielectrophoresis. If the particle is less polarisable than the surrounding medium, the dipole will align counter to the field and the particle will be repelled from the high field regions, called negative dielectrophoresis. 


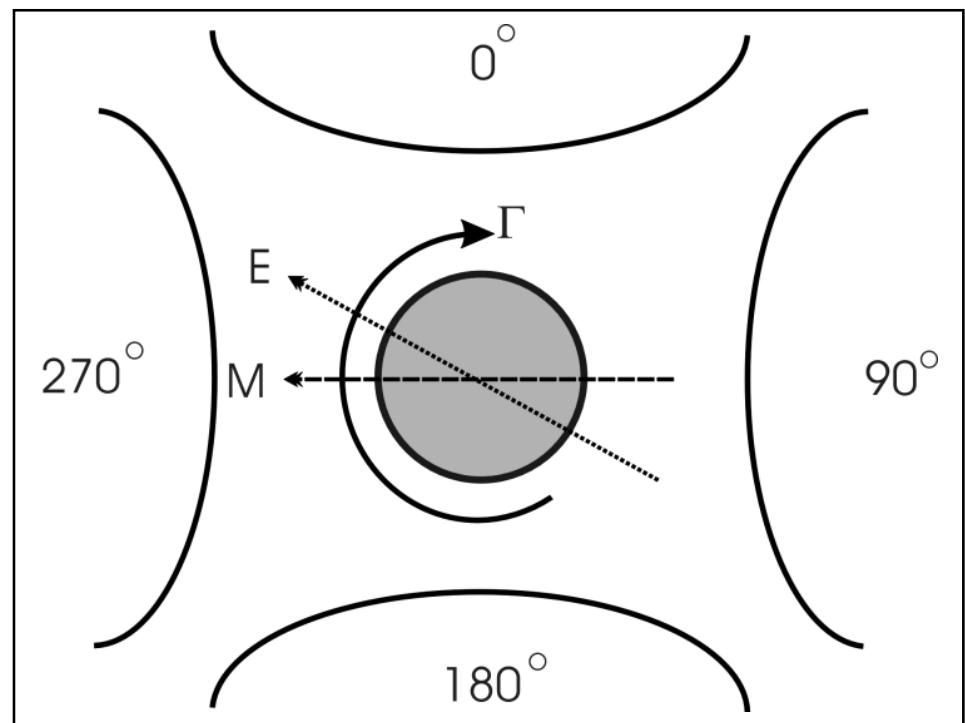

Figure 2. A schematic of a polarisable particle suspended in a rotating electric field generated by four electrodes with $90^{\circ}$ advancing phase. If the electric field E rotates sufficiently quickly, the induced dipole $\mathrm{M}$ will lag behind the electric field by an angle related to the time taken for the dipole to form (the relaxation time). The interaction between the electric field and the lagging dipole induces a torque $\square$ in the particle, causing the particle to rotate. This effect is known as electrorotation. 


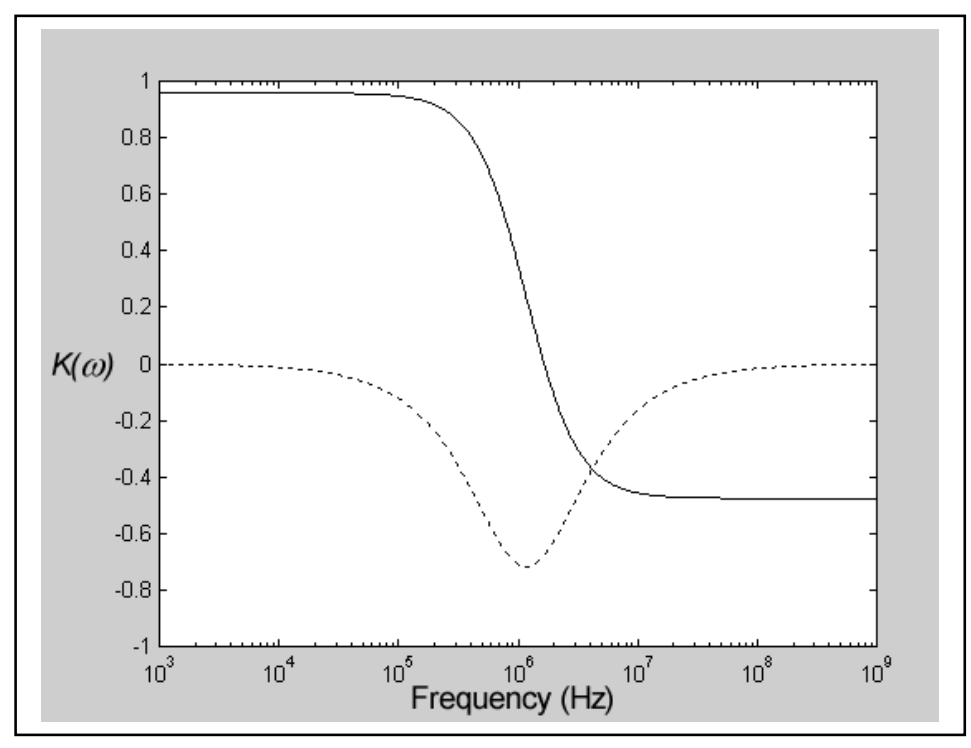

Figure 3. A plot of the real (solid line) and imaginary (dotted line) parts of the Clausius-Mossotti factor. The magnitude and signs of the real and imaginary parts govern the magnitude and direction of the dielectrophoretic force and electrorotational torque respectively. 


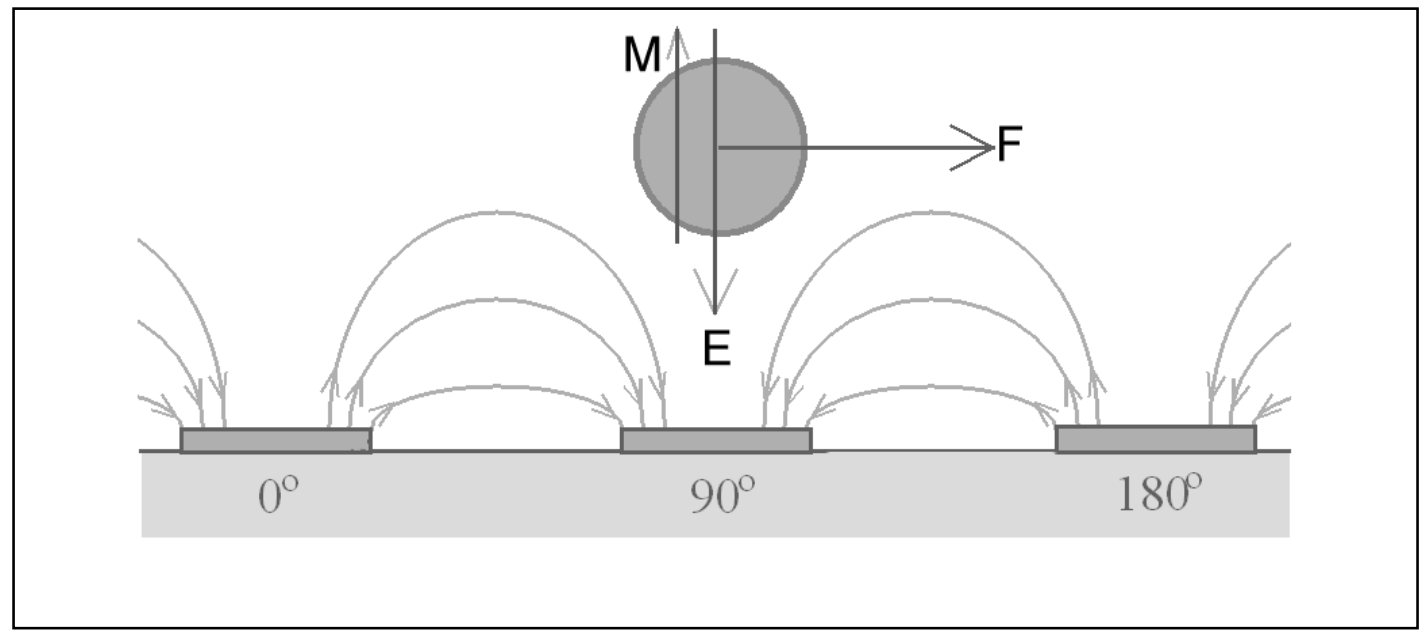

Figure 4. A schematic showing a polarisable particle suspended in a travelling electric field generated by electrodes on which the applied potential is $90^{\circ}$ phase-advanced with respect to the electrode to its left. If the electric field E moves sufficiently quickly, the induced dipole $\mathrm{M}$ will lag behind the electric field, inducing a force $\mathrm{F}$ in the particle. This causes the particle to move along the electrodes, a phenomenon known as travelling-wave dielectrophoresis. 


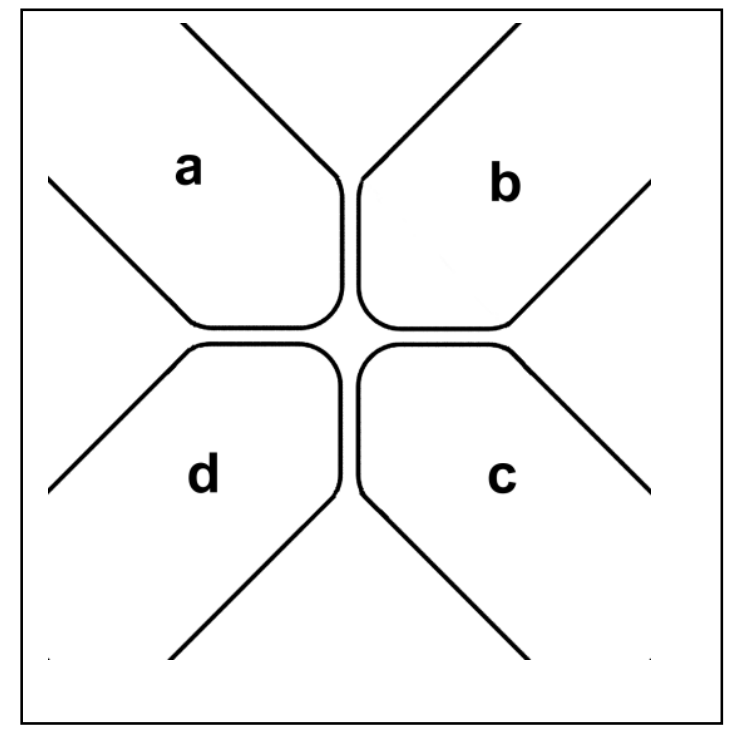

Figure 5. A schematic of typical quadrupole electrode microstructures used in dielectrophoresis experiments. The electrodes are typically fabricated of gold on a glass microscope slide. The gap between opposing electrodes in the centre of the array is typically of the order $10-50 \mu \mathrm{m}$ across, but can be as small as $500 \mathrm{~nm}$ or as large as $1 \mathrm{~mm}$. To induce dielectrophoretic motion in particles suspended near the electrode array, electrodes would be energised such that a and c are of the same phase, and $b$ and $d$ are in antiphase to them. To cause electrorotation in particles within the central gap, electrodes b, c and d would be phase-shifted by $90^{\circ}, 180^{\circ}$ and $270^{\circ}$ with respect to electrode a. 


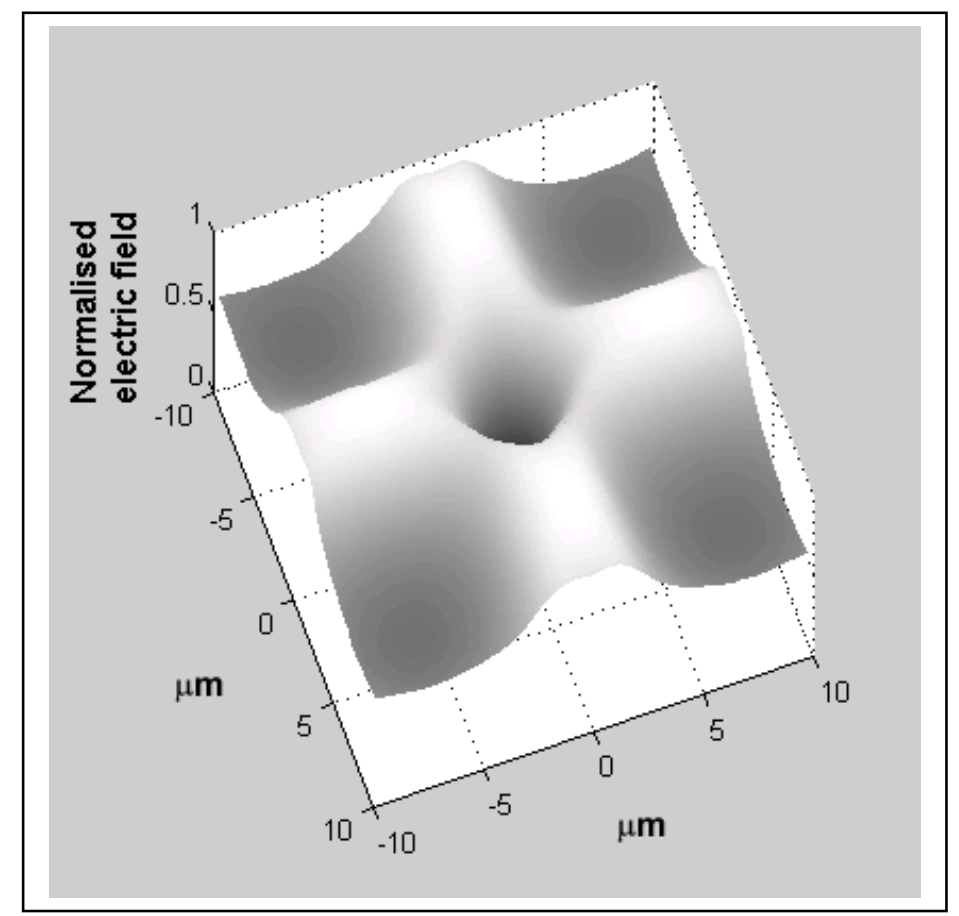

Figure 6. A simulation of the electric field in the plane $7 \mu \mathrm{m}$ above the electrode array shown in figure 4. The simulation was performed using a Method of Moments software suite developed by the author at the University of Wales at Bangor. As can be seen, there is an electric field minimum (dark region) at the centre of the electrode array, surrounded by a ring of high electric field gradient. Particles experiencing negative dielectrophoresis are repelled into this minimum and become trapped. The electric field strength is high (white regions) along the electrode edges, where particles experiencing positive dielectrophoresis will collect. 


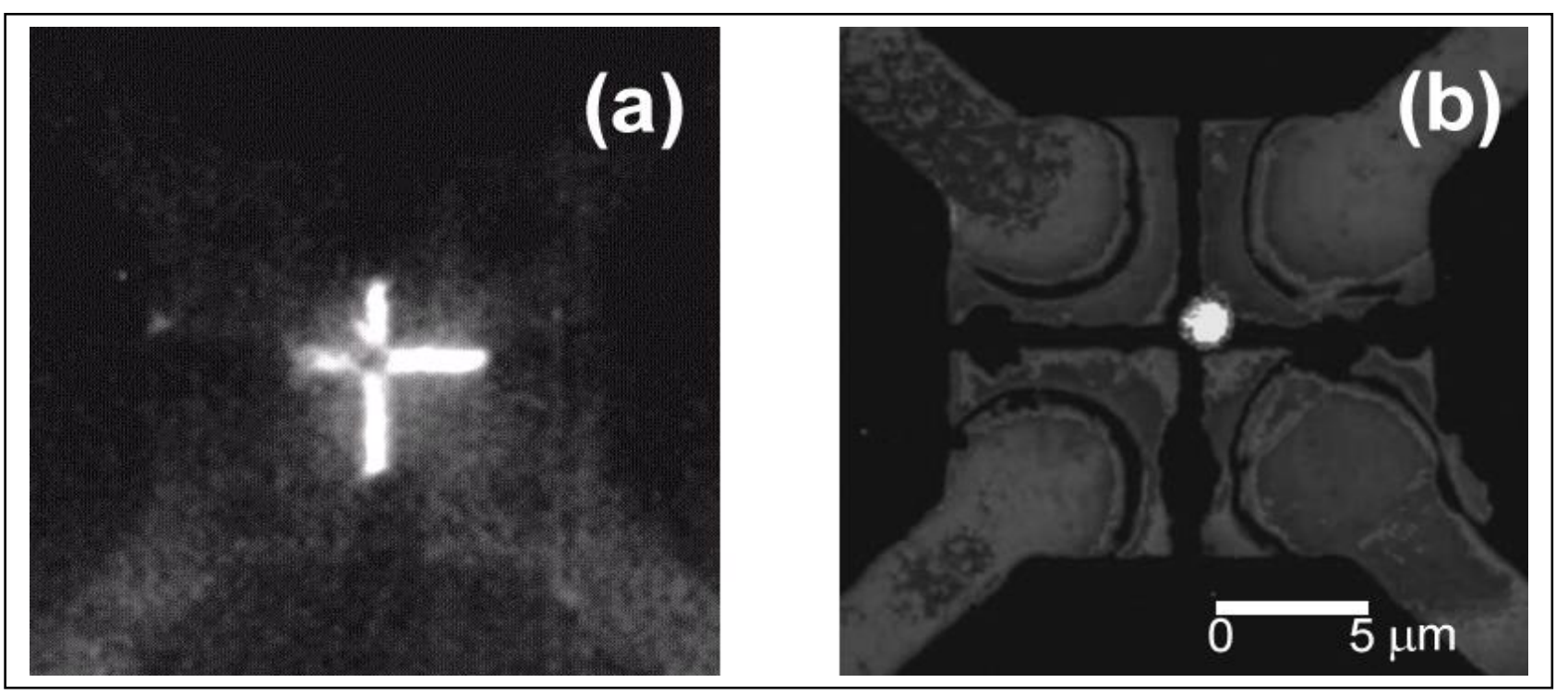

Figure 7. A photograph of $14 \mathrm{~nm}$ diameter, fluorescently labelled latex spheres precipitated from an aqueous solution (conductivity adjusted to $2.5 \mathrm{mSm}^{-1}$ by addition of $\mathrm{KCl}$ ) by dielectrophoresis. The electrodes were of similar construction to those shown in figure 5, with $500 \mathrm{~nm}$ between adjacent electrodes and $2 \square \mathrm{m}$ across the inter-electrode gap. In photograph (a), the spheres collect in the high-field regions between the electrode edges when a $8 \mathrm{~V}_{\mathrm{pp}}, 2 \mathrm{MHz}$ signal is applied. In photograph (b) the spheres collect in a ball at the centre of the trap, which is levitated a few $\square \mathrm{m}$ above the electrode plane when the applied signal is changed to $10 \mathrm{~V}_{\mathrm{pp}}, 10 \mathrm{MHz}$. Collection of particles is rapid and begins immediately after the field is applied. This experiment was performed at the Humboldt University of Berlin and first appeared in Müller et al. (1996); reproduced with permission. 


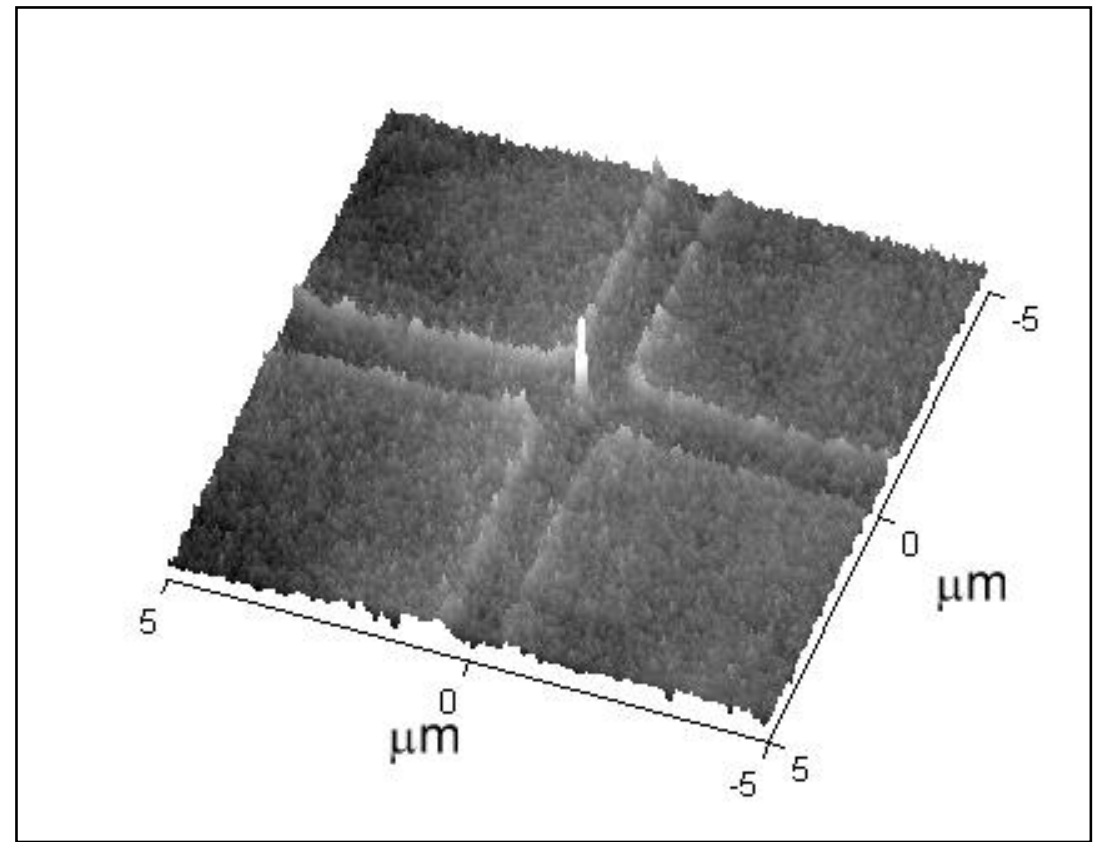

Figure 8. A pseudo-3D image of a single $93 \mathrm{~nm}$ latex sphere suspended in a trap by negative dielectrophoresis, using an electrode structure similar to that shown in figure 5 , with $6 \square \mathrm{m}$ between opposing electrode tips and $2 \square \mathrm{m}$ between adjacent electrodes. The medium conductivity was adjusted to $5 \mathrm{mSm}^{-1}$ by addition of EDTA, and the applied signal was $5 \mathrm{~V}_{\mathrm{pp}}, 15 \mathrm{MHz}$. The bead occupies the space at the point of the "spike" at the centre of the electrode structure. The vertical dimension was extracted from light emission data from fluorescence micrographs. This experiment was performed at the University of Glasgow and first appeared in Hughes and Morgan (1998); reproduced with permission. 
